\title{
Una mujer con delirio de parasitosis
}

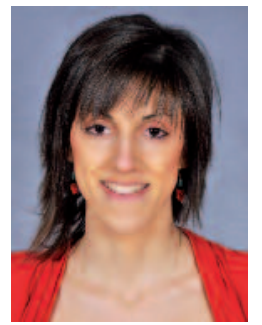

Elena Gil de la Cruz Residente de tercer año. Servicio de Dermatología Quirúrgica y Venereología. Hospital Universitario 12 de Octubre. Madrid.

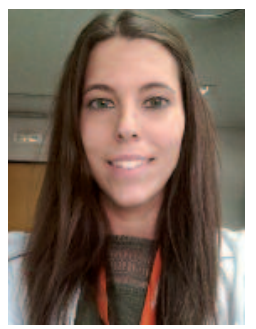

\section{Alba Calleja-Algarra}

Residente de segundo año. Servicio de Dermatología Quirúrgica y Venereología. Hospital Universitario 12 de Octubre. Madrid.

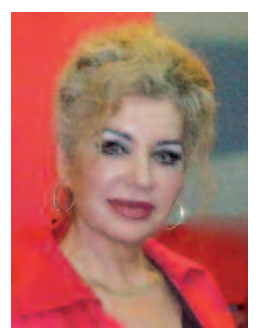

\section{Aurora Guerra-Tapia}

Jefa de sección del Servicio de Dermatología Quirúrgica y Venereología.

Hospital Universitario 12 de Octubre. Madrid.
Mi paciente es una mujer de 64 años que acudió a la consulta de dermatología por prurito en el cuero cabelludo desde hacía meses. Se mostraba desesperada, convencida de padecer una pediculosis refractaria a todos los tratamientos que había probado sin éxito.

Sin embargo, en la exhaustiva exploración realizada, no conseguimos detectar signo alguno de dicha enfermedad, al no observarse ningún parásito o liendre.

Ante su insistencia, se decidió programar una revisión al cabo de una semana, acudiendo entonces la paciente con un bote en el que había recogido lo que consideraba «la muestra» de su afección. De nuevo, la exploración del cuero cabelludo fue normal y, en el citado bote, tan solo pudimos reconocer algunos restos de escamas córneas. Ella, no obstante, se mostraba obcecada: «tenía que haber algo más». La muestra fue remitida al Departamento de Anatomía Patológica, en cuyo informe se describían «tallos pilosos con fragmentos de queratina de carácter inespecífico, sin reconocimiento de parásitos» (figs. 1 y 2). En la siguiente consulta, se planteó la necesidad de recibir un tratamiento psiquiátrico, a lo que finalmente accedió la paciente.

El delirio de parasitosis, también conocido como síndrome de Ekbom, es un trastorno psiquiátrico en el que los pacientes tienen una idea falsa y fija de estar infestados por parásitos, como ácaros, liendres, pulgas, arañas, gusanos u otros organismos.

Fue descrito por George Thibiérge en 1894 con el nombre de acarofobia, término que sería sustituido por neurodermatitis parasitofóbica en 1896 por Perrin, quien documentó tres casos. El neurólogo sueco Karl Axel Ekbom, en 1938, fue el primero en describir las causas, la sintomatología y el pronóstico de este cuadro, que bautizó como delirio presenil parasitario dermatozoico. Finalmente, Wilson y Miller, en 1946, lo denominaron delirio de parasitosis, con el nombre de sindrome de Ekbom como epónimo. 


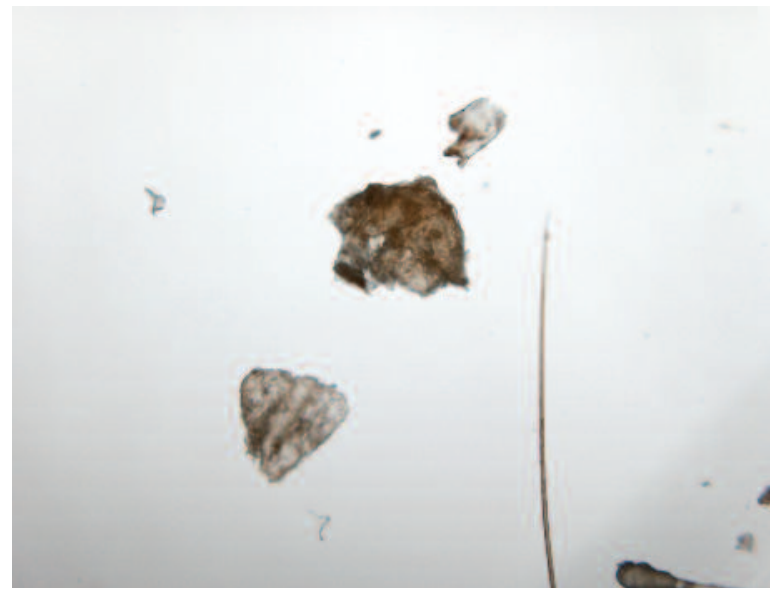

Figura 1. Tallos pilosos con fragmentos de queratina y escamas córneas.

Los pacientes tienen ideas elaboradas y detalladas acerca de los parásitos por los que creen estar infestados y presentan supuestas muestras de estos en papel, cinta adhesiva, bolsas, frascos, pequeñas cajas e, incluso, fotos.

Se trata de un subtipo de cuadro delirante incluido dentro de los de tipo somático. En estos, aparecen en el paciente ideas delirantes de que tiene algún defecto físico o una enfermedad médica, en ausencia de evidencias objetivas, malinterpretando las sensaciones subjetivas corporales. Clásicamente, se han distinguido dos tipos: el ectoparasitario, en el que la invasión se circunscribe a la piel y que es, por lo tanto, el que llega a la consulta de dermatología, como el de nuestra paciente; y el endoparasitario, cuando afecta a orificios u órganos internos (generalmente, al tubo digestivo).

Aunque puede ocurrir a cualquier edad, se observa una distribución bimodal, con predominio en el género femenino, que aumenta con la edad ${ }^{1}$. Es frecuente que sean personas con pocos contactos sociales o familiares, sin historia de enfermedades psiquiátricas y funciones cognitivas normales, oscilando la duración media del delirio, según las series, entre los 9 y los 18 meses. Se estima que entre el 5 y el $15 \%$ de ellos (Trabert, 1999) está asociado a un trastorno psicótico compartido o folie à deux. Se trata de pacientes que trans-

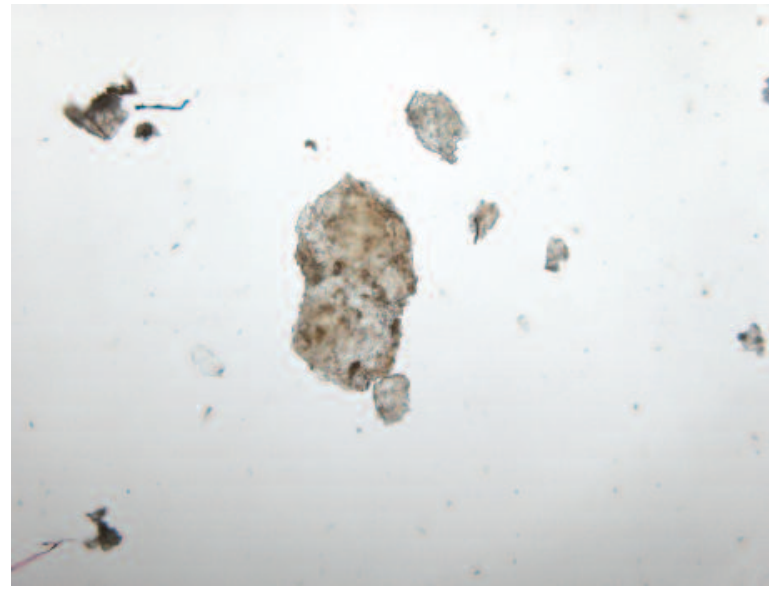

Figura 2. Escamas córneas aisladas.

miten el delirio a personas cercanas, frecuentemente, algún familiar, lo que complica el tratamiento y la curación del trastorno ${ }^{2}$. En nuestro caso, no obstante, no se produjo este fenómeno.

Las causas y la fisiopatología de este síndrome son poco conocidas. Existen teorías que sugieren que podría ser causado por mecanismos biológicos cerebrales (recientemente, se encontró una probable relación con el gen $H L A-{ }^{*} 03$ ), factores epidemiológicos como la edad avanzada, antecedentes familiares o alteración de la personalidad y la percepción. En cuanto a su posible origen psicógeno, se han postulado distintas hipótesis. Así, algunos autores consideran que se trata de una idea delirante primaria o una alteración del pensamiento. Otros, en cambio, señalan que lo primario es una alteración de la percepción y que el delirio se produce secundariamente a ella; esto es, constituye una elaboración delirante más o menos estructurada. También se ha dicho que es probable que ambos mecanismos coexistan ${ }^{3}$.

El motivo de consulta principal es el prurito cutáneo asociado a excoriaciones, observándose en la exploración física únicamente las lesiones secundarias a este. La topografía de los signos cutáneos corresponde a lugares que se encuentran al alcance de la mano del paciente y, habitualmente, es asimétrica, con predominio de uno u otro 
lado según el miembro dominante. En nuestra paciente, al referir el prurito en el cuero cabelludo, tenía ligera descamación secundaria al rascado que seguía una distribución bastante homogénea.

Lo habitual es que los enfermos acudan al médico con los patógenos imaginarios ficticios que ellos han capturado en su propia piel en frascos, cajas o envueltos en plástico y papel, solicitando un estudio microscópico de los «parásitos». Este rasgo, presente en el caso expuesto y conocido como «signo de la caja de cerillas», es patognomónico del trastorno.

El proceso diagnóstico conlleva una historia clínica detallada y una exploración física minuciosa, pudiendo ser necesarios, además, diversos estudios de laboratorio, biopsia cutánea y análisis de muestras aportadas por el paciente. Es necesario descartar infestaciones como la escabiosis, la pediculosis y la miasis, así como causas de prurito metabólico. El diagnóstico diferencial, además de con verdaderas parasitosis, debe hacerse con enfermedades psiquiátricas que pueden simular este delirio, como las alucinaciones de la esquizofrenia o la depresión, así como con el delirio de parasitosis inducido por fármacos para el tratamiento de la enfermedad de Parkinson o secundario a la toma de cocaína (delirio de Magnan).

El abordaje de estos pacientes es difícil, ya que cualquier insinuación sobre la etiología psiquiátrica del trastorno es razón suficiente para que abandonen el seguimiento. El dermatólogo, además de tratar las lesiones de la piel, debe iniciar la terapia con antihistamínicos orales durante la primera consulta para el tratamiento del prurito cutáneo mientras se esperan los resultados de los estudios de laboratorio adicionales para investigar otras causas de prurito o parasitosis reales.

El tratamiento farmacológico consiste en la administración de antipsicóticos. Clásicamente, se ha observado una respuesta buena y específica al antipsicótico típico pimozida e, incluso, se seguía recomendando en artículos recientes; pero este no es el tratamiento de primera línea, por su perfil de efectos secundarios. En los últimos años, varios artículos han indicado los efectos beneficiosos de algunos antipsicóticos atípicos, como la risperidona, la quetiapina, la amisulprida y olanzapina ${ }^{1}$.

La mayoría de estos pacientes se pierden durante el seguimiento, pero, entre los que aceptan el tratamiento, se describe una evolución favorable en el $80 \%$ de los casos. El pronóstico mejora considerablemente cuanto más corto sea el período sintomático previo al tratamiento. Es muy importante que el individuo acepte la participación de la psiquiatría en su tratamiento, lo cual debe darse después de algunas citas de evaluación, en las que se evite discutir la causa de la enfermedad y se haya establecido una relación basada en la confianza entre el dermatólogo y el paciente ${ }^{4,5}$.

\section{BIBLIOGRAFÍA}

1. Zamora Rodríguez FJ, Benítez Vega C, Calderón López P, Guisado Macías JA, Teva García MI, Sánchez-Waisen Hernández MR. Un caso de síndrome de Ekbom. Psiquiatr Biol. 2011;18(1):42-4.

2. Viejo JL, Sánchez C. Una infestación parasitaria por insectos ficticia. Descripción de un caso de síndrome de Ekbom. Bol R Soc Esp Hist Nat Sec Biol. 2010;104(1-4):47-9.

3. Moreno VK, Ponce ORM, Narváez RV, Ubbelohde HT. Síndrome de Ekbom. Dermatología Rev Mex. 2007;51(2):51-6.

4. Viejo Montesinos JL. Dermatología y entomología. Más Dermatol. 2011;14:2-4.

5. Guerra-Tapia A. Dermatología psiquiátrica: de la mente a la 